\title{
Half-cycle QAM modulation for VCSEL-based optical links
}

Pham, Tien Thang; Rodes Lopez, Roberto; Jensen, Jesper Bevensee; Chang-Hasnain, J.; Tafur Monroy, Idelfonso

Published in:

ECOC Technical Digest

Link to article, DOI:

10.1364/ECEOC.2012.Mo.1.B.3

Publication date:

2012

Document Version

Publisher's PDF, also known as Version of record

Link back to DTU Orbit

Citation (APA):

Pham, T. T., Rodes Lopez, R., Jensen, J. B., Chang-Hasnain, J., \& Tafur Monroy, I. (2012). Half-cycle QAM modulation for VCSEL-based optical links. In ECOC Technical Digest (pp. Mo.1.B.3). Optical Society of America. https://doi.org/10.1364/ECEOC.2012.Mo.1.B.3

\section{General rights}

Copyright and moral rights for the publications made accessible in the public portal are retained by the authors and/or other copyright owners and it is a condition of accessing publications that users recognise and abide by the legal requirements associated with these rights.

- Users may download and print one copy of any publication from the public portal for the purpose of private study or research.

- You may not further distribute the material or use it for any profit-making activity or commercial gain

- You may freely distribute the URL identifying the publication in the public portal 


\title{
Half-cycle QAM modulation for VCSEL-based optical links
}

Tien-Thang Pham ${ }^{1}$, Roberto Rodes ${ }^{1}$, Jesper Bevensee Jensen ${ }^{1}$, Connie. J. Chang-Hasnain ${ }^{2}$ and Idelfonso Tafur Monroy ${ }^{1}$

(1) DTU Fotonik, Department of Photonics Engineering, Technical University of Denmark, DK2800 Kgs.

Lyngby, Denmark, ptit@fotonik.dtu.dk

(2) Dept. of Electrical Engineering and Computer Science, University of California, Berkeley, CA

94720, USA, cch@eecs.berkeley.edu

\begin{abstract}
Novel spectrally efficient half-cycle QAM modulation is experimentally demonstrated. 10 Gbps 4-QAM signal in 7.5-GHz bandwidth was successfully transmitted over $20 \mathrm{~km}$ SMF using an uncooled $1.5 \mu \mathrm{m}$ VCSEL with no equalization applied.
\end{abstract}

\section{Introduction}

Vertical cavity surface emitting lasers (VCSELs) have several attractive properties such as large modulation bandwidth, low driving voltage, wavelength tunability, wafer-scale testing, easy packaging, and low carbon footprint [1-4]. These advantages make VCSELs attractive light sources for high-speed optical communication links in data centers and optical access networks. Optical links that use long-wavelength VCSELS and on-off keying (OOK) modulation are limited in transmission reach by chromatic dispersion of optical fibers due to frequency chirping caused by direct modulation of the VCSEL and large occupied bandwidth of signals [5-8]. For 10-Gbps data transmission using 1.5$\mu \mathrm{m}$ VCSELs, 3-dB power penalty after 10-km single mode fiber (SMF) was experimentally observed [6] and 11-dB power penalty after 20$\mathrm{km}$ SMF was simulated [7]. To deal with the effect of chromatic dispersion for OOK systems, dispersion management and dispersion mitigation methods have been proposed [5-8]. Advanced modulation formats also have been proposed to reduce the bandwidth of the signals. Multi-tone modulation (DMT), a subclass of orthogonal frequency division multiplexing (OFDM), is a promising technology for both increasing spectral efficiency and dispersion tolerance. High speed data transmission of DMT signal using VCSELs has been demonstrated recently [9-10]. However, to support high-speed data transmission, DMT requires fast analogue to digital (ADC) and digital to analogue (DAC) converters. Additionally, DMT transceivers consume high power for signal processing compared to conventional OOK transceivers. A simpler method, single-cycle subcarrier QAM modulation was proposed in which the subcarrier frequency is equal to the baud rate. Transmission of 10 Gbaud 16-QAM data in 20$\mathrm{GHz}$ bandwidth over 200-m MMF using 850-nm
VCSELs has been reported [11].

In this paper, we demonstrate half-cycle QAM modulation for spectrally efficient VCSEL-based optical links. By using a subcarrier frequency at a half the baud rate, the spectrum of the QAM signal is reduced $25 \%$ compared to conventional and single-cycle QAM modulation. We report the transmission of real-time generated $10-\mathrm{Gpbs}$ and 16-Gbps 4-QAM signals over $20-\mathrm{km}$ and 3$\mathrm{km}$ SMFs respectively using an un-cooled commercially available $10-\mathrm{GHz} 1.5-\mu \mathrm{m}$ VCSEL. Bit error rate (BER) below the limit of the forward error correction (FEC) with 7\% payload overhead was achieved for both cases. Only 2.5 dB power penalty was observed for 10 Gbps 4QAM signal after 20-km fiber transmission.

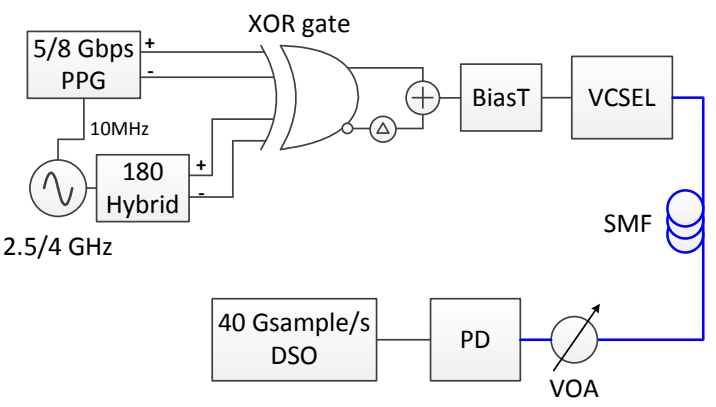

Fig. 1: Experimental setup: Pulse pattern generator (PPG), photodetector (PD), variable optical attenuator (VOA), single mode fiber (SMF), digital storage oscilloscope (DSO).

\section{Experimental setup}

Fig. 1 shows the experimental setup. There are two important parts in the setup: real time generation and transmission of a half-cycle 4QAM signal.

a) Half-cycle 4-QAM signal generation

To generate the electrical half-cycle 4-QAM signal, we used the method proposed in [8]. However, in our case, the subcarrier frequency was equal to half the bit rate. $2.5 / 4-\mathrm{GHz}$ subcarrier and synchronized 5/8-Gbps data with PRBS length of $2^{10}-1$ were fed to a $13-\mathrm{GHz}$ 
bandwidth XOR gate (Inphi 13610XR) to create two BPSK signals in the two outputs of the XOR gate. One output of the XOR gate was delayed and then combined with the other one using a power combiner to form a 4-QAM signal. The delay time was optimized in order to secure that the two signals from the XOR outputs are uncorrelated and 90 degrees out of phase.

The half-cycle QAM signal has some special features. Firstly, the first null in the spectrum of the signal is at 1.5 times the symbol rate while the first null point of single cycle QAM modulation is at twice the symbol rate. This means that the spectral efficiency of half-cycle QAM modulation is improved by $25 \%$ compared to single cycle modulation. The spectra of the generated half-cycle and single-cycle 5-Gbaud 4-QAM signals are illustrated in Fig. 2. It is observed that the width of the first lobe of halfcycle modulation and single-cycle modulation is 7.5 and $10 \mathrm{GHz}$, respectively. Secondly, unlike the single-cycle QAM signal, most of the power of the half-cycle QAM signal is concentrated in the low frequency region. This makes the signal more tolerant towards high-frequency roll-off of VCSELS and photodiodes.

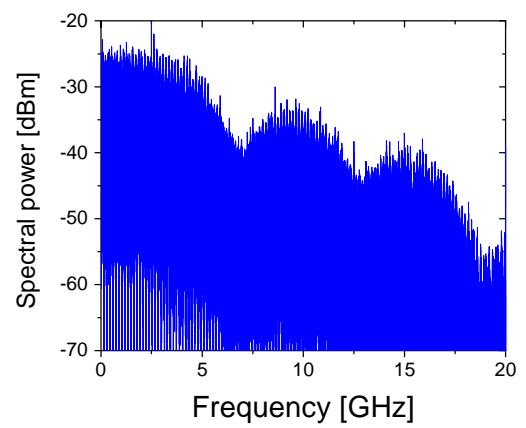

a)

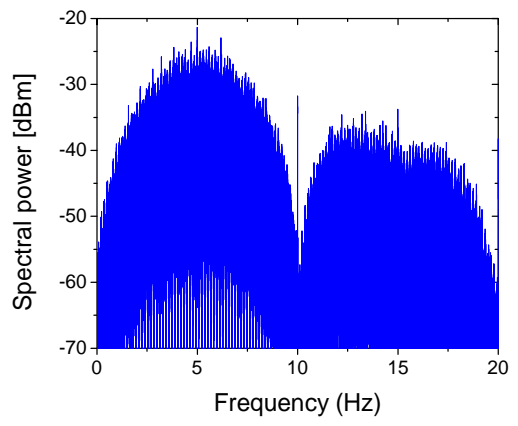

b)

Fig.2: Spectrum of a) 5-Gbaud half-cycle 4-QAM signal and b) 5-Gbaud single-cycle 4-QAM signal

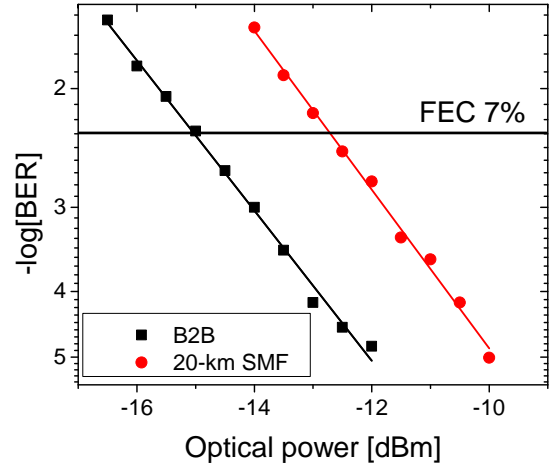

a)

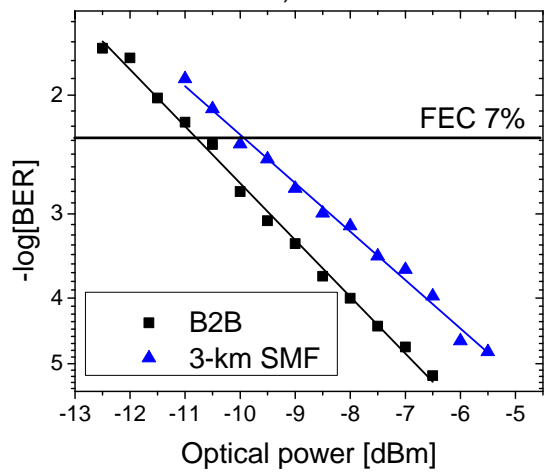

b)

Fig.3: Performance of 4-QAM signals at B2B and after fiber transmission a) 5 Gbaud, b) 8 Gbaud

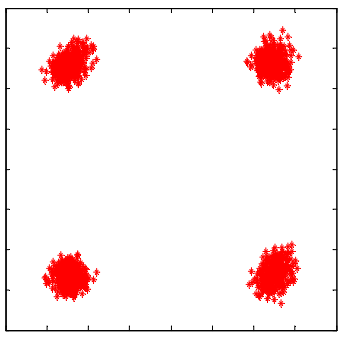

a)

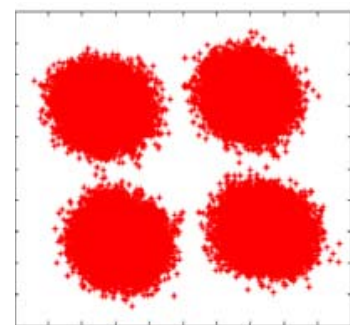

b)
Fig.4- Constellation of 5-Gbaud signal, a) generated electrical signal and b) at $-12.0 \mathrm{dBm}$ B2B

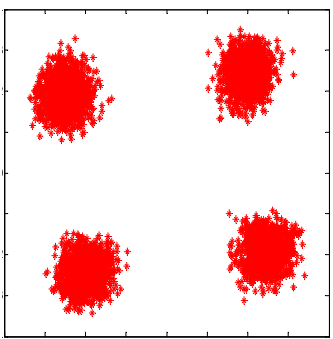

a)

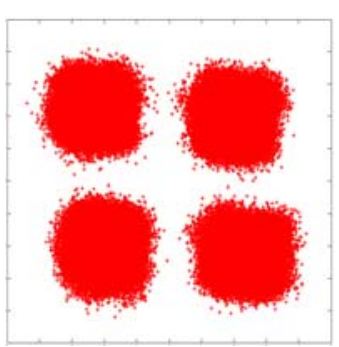

b)
Fig. 5. Constellation of 8-Gbaud signal, a) generated electrical signal and $b$ ) at $-6.5 \mathrm{dBm}$ B2B

b) Half cycle QAM signal transmission The generated 4-QAM signal was fed to an uncooled 1.5- $\mu \mathrm{m}$ VCSEL using a BiasT. The threshold current of the VCSEL was $17 \mathrm{~mA}$ and 
it is biased at $22.5 \mathrm{~mA}$ for the best performance. With 5-Gbaud data, the optical signal from the VCSEL was transmitted over $20-\mathrm{km}$ standard SMF. The 8-Gbaud signal was transmitted through 3-km SMF. A variable optical attenuator (VOA) was utilized to vary the optical power level into the photodetector (PD). The 3-dB bandwidth of the VCSEL, evaluation board and PD was approximately $10 \mathrm{GHz}$. The photodetected signal was digitized using a 40GSamples/s digital storage oscilloscope (DSO) for offline digital signal processing (DSP. The DSP algorithm was kept simple without any equalization technique. It includes clock recovery, I/Q detection, threshold detection and bit error rate (BER) calculation.

\section{Experimental results}

To assess the performance of the system, approximately 150k symbols (300k bits) were used to calculate the BER of the signals for both data rates. Fig. 3 shows the BER of the signals B2B and after fiber transmission. For the 5Gbaud signal, there were no errors detected when the optical power was below $-12 \mathrm{dBm}$ and bellow $-10 \mathrm{dBm}$ for $\mathrm{B} 2 \mathrm{~B}$ and fiber transmission cases, respectively. Assuming that $7 \%$ payload is used for FEC header, the limit of pre-FEC BER aiming for after- FEC BER of $10^{-15}$ is $4.8 \times 10^{-3}$ [12]. At the FEC limit, the receiver sensitivity B2B was approximately $-15 \mathrm{dBm}$ and the power penalty after 20-km SSMF transmission was only $2.5 \mathrm{~dB}$.

For 8 Gbaud data B2B, as shown in Fig. 3.b, there were no errors detected at $-6.0 \mathrm{dBm}$ and the receiver sensitivity at the FEC limit was $11.0 \mathrm{dBm}$. Due to the increased bandwidth, the 8-Gbaud signal has higher transmission power penalty than the 5-Gbaud signal. After 3-km SMF, the power penalty was approximately 1.5 $\mathrm{dB}$. In general, the receiver sensitivity was approximately $5 \mathrm{~dB}$ worse than the 5-Gbaud signal. This performance limitation are attributed the imperfection of the generated QAM signal before modulation and limited bandwidth of the transceivers. The generated $8 \mathrm{Gbaud}$ signal had lower signal-to-noise ratio (SNR) than the 5Gbaud signal and the bandwidth of the 8-Gbaud signal was $12 \mathrm{GHz}$. Examples of the constellations of both 5 Gbaud and 8Gbaud signals before modulating the VCSEL and at B2B are presented in Fig. 4 and Fig. 5.

\section{Conclusions}

We have investigated half-cycle subcarrier QAM modulation for VCSEL-based optical links with $25 \%$ better spectral efficiency than other QAM modulation. 10-Gbps data in $7.5-\mathrm{GHz}$ bandwidth was transmitted through $20-\mathrm{km}$ single mode fiber with BER below the FEC limit and only 2.5$\mathrm{dB}$ power penalty. Half-cycle subcarrier QAM modulation has potential for applications in simple, spectrally efficient high-speed PON networks as well as high performance data centers.

\section{Acknowledgements}

The authors would also like to thank $\mathrm{C}$. Neumeyr, E. Rnneberg and M. Ortsiefer from Vertilas $\mathrm{GmbH}$ for supplying high-quality VCSELS.

\section{References}

[1] W. Hofmann, Electronics Letters, 47, 270 (2011).

[2] P. Moser, et al., Proc. ECOC, (2011).

[3] W. Hofmann, et al., Proc. CLEO/QELS 2008, pp.1- 2, (2008).

[4] M. C. Y. Huang, IEEE J. Selected Topics in Quantum Electronics, 13, 374 (2007).

[5] B.Zhang, in Proc. OFC, OWT7 (2008).

[6] L. Xu, et al., Proc. CLEO-EQEC (2009).

[7] T. B. Gibbon, et al., Optical Fiber Tech., 17, 41, (2011).

[8] K. Prince, et al., IEEE/OSA JOCN, 3, 399, (2011).

[9] E. Hugues- Salas et al,. Optics Express, 19, 2979 (2011).

[10] S.C.J. Lee, et al,. Proc. OFC, PDP6, (2009).

[11] K. Szczerba et al., Proc. ECOC, We.7.B.2 (2011).

[12] J. Justesen, IEEE Trans. Commun. 59, 407 (2011). 